Article

\title{
End-To-End Deep Learning Architecture for Continuous Blood Pressure Estimation Using Attention Mechanism
}

\author{
Heesang Eom ${ }^{1,+}{ }^{\text {, Dongseok Lee }}{ }^{2,+} \oplus$, Seungwoo Han ${ }^{3}\left(\mathbb{D}\right.$, Yuli Sun Hariyani ${ }^{1,4} \oplus$, Yonggyu Lim ${ }^{5}$, \\ Illsoo Sohn ${ }^{6}$, Kwangsuk Park ${ }^{7,8, *}$ and Cheolsoo Park ${ }^{1, *}$ \\ 1 Department of Computer Engineering, Kwangwoon University, Seoul 01897, Korea; \\ surmounting@kw.ac.kr (H.E.); yulisun@telkomuniversity.ac.id (Y.S.H.) \\ 2 Interdisciplinary Program in Bioengineering, Seoul National University, Seoul 03080, Korea; \\ azuremoon@bmsil.snu.ac.kr \\ 3 Department of Intelligent Information System and Embedded Software Engineering, \\ Kwangwoon University, Seoul 01897, Korea; seungwoohan@kw.ac.kr \\ 4 School of Applied Science, Telkom University, Bandung 40257, Indonesia \\ 5 Department of Oriental Biomedical Engineering, Sangji University, Wonju 26339, Korea; yglim@sangji.ac.kr \\ 6 Department of Computer Science and Engineering, Seoul National University of Science and Technology, \\ Seoul 01811, Korea; isohn@seoultech.ac.kr \\ 7 Department of Biomedical Engineering, College of Medicine, Seoul National University, Seoul 03080, Korea \\ 8 Institute of Medical and Biological Engineering, Medical Research Center, Seoul National University, \\ Seoul 03080, Korea \\ * Correspondence: kwspark@snu.ac.kr (K.P.); parkcheolsoo@kw.ac.kr (C.P.) \\ + These authors contributed equally to this work.
}

Received: 11 March 2020; Accepted: 17 April 2020; Published: 20 April 2020

check for updates

\begin{abstract}
Blood pressure (BP) is a vital sign that provides fundamental health information regarding patients. Continuous BP monitoring is important for patients with hypertension. Various studies have proposed cuff-less BP monitoring methods using pulse transit time. We propose an end-to-end deep learning architecture using only raw signals without the process of extracting features to improve the BP estimation performance using the attention mechanism. The proposed model consisted of a convolutional neural network, a bidirectional gated recurrent unit, and an attention mechanism. The model was trained by a calibration-based method, using the data of each subject. The performance of the model was compared to the model that used each combination of the three signals, and the model with the attention mechanism showed better performance than other state-of-the-art methods, including conventional linear regression method using pulse transit time (PTT). A total of 15 subjects were recruited, and electrocardiogram, ballistocardiogram, and photoplethysmogram levels were measured. The 95\% confidence interval of the reference BP was [86.34, 143.74] and [51.28, 88.74] for systolic BP (SBP) and diastolic BP (DBP), respectively. The $R^{2}$ values were 0.52 and 0.49 , and the mean-absolute-error values were $4.06 \pm 4.04$ and $3.33 \pm 3.42$ for SBP and DBP, respectively. In addition, the results complied with global standards. The results show the applicability of the proposed model as an analytical metric for BP estimation.
\end{abstract}

Keywords: blood pressure; electrocardiogram; photoplethysmogram; ballistocardiogram; deep learning; signal processing; attention mechanism

\section{Introduction}

Blood pressure (BP) is one of the vital signs that provide fundamental health information of the patient. When the heart beats, the blood flow from the heart exerts a pressure on the blood vessels. 
BP varies between systolic $\mathrm{BP}$ (SBP), which is an increase in pressure caused by systolic contraction of the heart, and diastolic BP (DBP), which is the low pressure between SBPs. High BP (hypertension) is known as a "silent killer" because it is a risk factor for various diseases such as arrhythmia, heart attack, blindness, and brain stroke. An estimated 1.13 billion people worldwide have hypertension [1].

The gold standard of BP monitoring is a sphygmomanometer, which is usually used in the physician's office. However, BP measurement in medical circumstances may be incorrect because, in a clinical setting, some patients exhibit a higher BP than usual, which is known as the "white-coat effect." The arterial BP (ABP) is considered as a gold standard for continuous BP monitoring, which is performed in the intensive care unit. However, measurement of ABP can cause side effects such as bleeding and infection because it is an invasive method that requires the insertion of an intravascular cannula needle. Moreover, it is difficult to measure ABP in daily life because it requires a clinical setting.

Because regular BP monitoring is important for the diagnosis of hypertension and the prediction of heart diseases, numerous devices have been created to help patients measure BP at home or during their daily lives. These devices are usually based on the oscillometric method and use an inflatable upper-arm cuff [2,3]. However, these methods do not offer continuous measurement. Currently, the significance of beat-to-beat BP analysis has increased, such as blood pressure variability (BPV) [4]. In addition, wearing the cuff whenever the patient tries to measure BP is a cumbersome task and can make the patient feel uncomfortable.

To overcome these limitations, several researchers have investigated cuff-less and continuous BP monitoring methods using physiological signals. The BP can be estimated based on the pulse wave velocity (PWV), which is the velocity of the arterial blood wave [5]. PWV can be calculated from the pulse transit time (PTT) as follows:

$$
P W V=L / P T T
$$

where $\mathrm{L}$ denotes the distance between two places where the blood wave propagates and PTT is the time that the blood wave takes to travel between two places.

The most frequently used definition of PTT is the time interval between the R peak of the electrocardiogram (ECG) and the systolic peak of the photoplethysmogram (PPG), which is measured at the finger. PTT from ECG and PPG can be easily applied in long-term monitoring or daily life situations because both ECG and PPG are measured noninvasively and do not require a medical expert or a cuff. PTT is known to be negatively correlated with BP [6], and various models have been developed to estimate BP using PTT [5,7-10].

In addition, some studies have suggested ballistocardiograms (BCGs) to estimate BP [11]. A BCG is a measurement of the forces of the body to the blood flow ejected from the heart after each heartbeat. BCG signals can be acquired using force sensors such as an accelerometer, load-cell, and polyvinylidene fluoride (PVDF) film sensor. This method enables unobtrusive BP estimation because BCG is a noninvasive and unconstrained method to monitor cardiovascular activity. Shin et al. suggested a system for BP estimation using a weighing scale [12]. ECG and BCG were measured on a weighing scale, and the R-J interval (RJI) was calculated between the R peak of the ECG and the J peak of the BCG signal. The results showed that RJI was negatively correlated with BP. Lee et al. proposed a chair-based BP monitoring system using a two channel BCG [13]. BP was estimated using the phase difference between two BCGs measured at the back of the chair and the cushion on the seat.

Other studies have proposed using machine learning (including deep learning) algorithms to estimate continuous BP without a cuff automatically. Chan et al. [14] and Kachuee et al. [15] proposed a model for estimating BP based on features extracted from ECG and PPG using conventional machine learning algorithms such as linear regression and AdaBoost. Su et al. proposed an recurrent neural network (RNN) based BP estimation model using features extracted from ECG and PPG [16]. Kurylyak et al. [17], Lee et al. [13], and Wang et al. [18] also proposed a simple artificial neural network (ANN) model that used features extracted from only a single signal, such as PPG or BCG. However, these methods still have problems that the extraction of features is costly and a laborious 
task. In addition, if the signal is noisy, it may be difficult to obtain enough data to train the neural network.

In recent studies, some authors have attempted to estimate BP using raw signals without feature engineering. Slapnivcar et al. estimated BP with only raw PPG using ResNet, a deep learning model that performed well in the field of image classification [19] but had lower performance compared to the models used in other studies. Tanveer et al. also used ECG and PPG raw signals and achieved good performance [20]. However, the length of the data used for BP estimation was very long, $16 \mathrm{~s}$, and, accordingly, the number of samples used for the overall estimation was small, making it difficult to evaluate performance accurately.

In this study, we developed an end-to-end deep learning model for BP estimation without feature extraction and used three physiological signals (ECG, PPG, and BCG) that were used in previous studies for estimating BP. The advantages of this study are as follows:

- $\quad$ BP can be estimated using only raw signals with minimal preprocessing.

- All combinations of signals were used as input, and their performance studied.

- By using the attention mechanism, the performance of the model was improved and its applicability as an analytical metric for BP estimation verified.

\section{Materials and Methods}

The overall experiment process is shown in Figure 1. The details of each step are explained in the following sections.
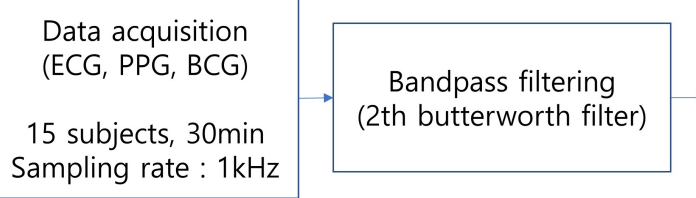

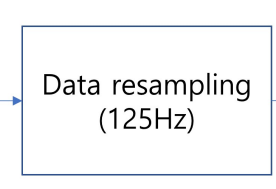

Deep learning

$(C N N+B i-G R U$

+ Attention)

Figure 1. Brief flow chart of our experiment.

\subsection{Data Acquisition}

A total of 15 subjects ( 6 men, 9 women, age: $26.2 \pm 3.0$ ) were recruited for this study. No medical records of the subjects were reported. Written informed consent was obtained from the subjects, and this study was approved by the Institutional Review Board of Seoul National University Hospital (IRB No. 1801-016-912).

The experimental setup is illustrated in Figure 2. Three $\mathrm{Ag} / \mathrm{AgCl}$ electrodes were attached to the subject's left arm, right arm, and left leg, according to Einthoven's triangle. ECG was acquired on lead II using the electrodes with the BIOPAC ECG100C module, and PPG was measured at the subject's index finger using a commercial module (PSL-iPPG2C) [21]. In addition, the BCG signals were measured using a PVDF film sensor attached on the seat of the chair. SBP and DBP were also measured simultaneously using a continuous BP monitoring device (Finometer Pro (Finapres Medical Systems, Enschede, The Netherlands)). All the data were synchronized and sampled at $1000 \mathrm{~Hz}$ with a data acquisition device (BIOPAC MP150 module (BIOPAC Systems Inc., Goleta, CA, USA)). 


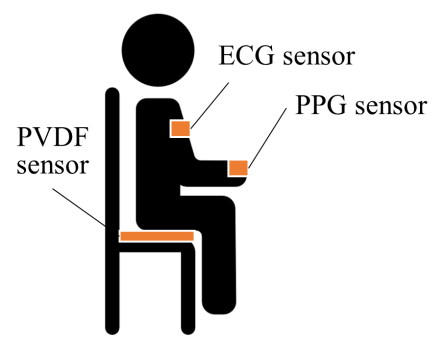

Figure 2. Overview of our experimental setup.

After the measurement device was attached, the subjects were asked to sit in the armchair. The data were measured after $30 \mathrm{~min}$, while the subjects rested. The measured BP values are shown in Figure 3. The mean and standard deviation (SD) of SBP and DBP were $115.04 \pm 14.64 \mathrm{mmHg}$ and $70.01 \pm 9.56 \mathrm{mmHg}$, respectively.

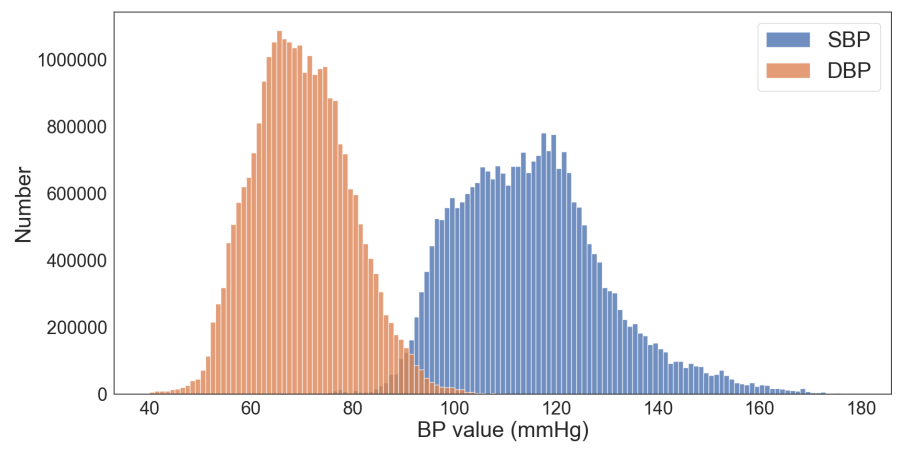

Figure 3. Distribution of BP values in the dataset used in this study.

\subsection{Data Preprocessing}

A second-order Butterworth bandpass filter was applied to the data to remove baseline wandering and power-line noise. The cutoff frequency of each filter applied to each signal is summarized in Table 1.

Table 1. The cutoff frequency of the filter applied to each signal.

\begin{tabular}{ccc}
\hline Signal & HPF $\mathbf{( H z )}$ & LPF $\mathbf{( H z )}$ \\
\hline ECG & 0.5 & 35 \\
BCG & 4 & 15 \\
PPG & 0.5 & 15 \\
\hline
\end{tabular}

All combinations of the raw signals (ECG, PPG, and BCG) were used as input for the deep learning model to investigate the effect of each signal on BP estimation. To ensure sufficient information was provided as input, $5 \mathrm{~s}$ segments of the signals were used as input to the model with an overlap of $1 \mathrm{~ms}$. Since a large amount of data were used as input, the data were resampled to $125 \mathrm{~Hz}$ for efficient learning, which is equivalent to the sampling rate of the Physionet MIMIC database containing ECG and PPG signals [22]. The sampling rate of the BCG signal was also resampled to $125 \mathrm{~Hz}$ with reference to [23]. The target labels were set as BP values (SBP, DBP) corresponding to the end of each segment, as shown in Figure 4. The BP values that were not in the range between the mean $\pm 1.96 \mathrm{SD}$ were considered as outliers and were eliminated. 

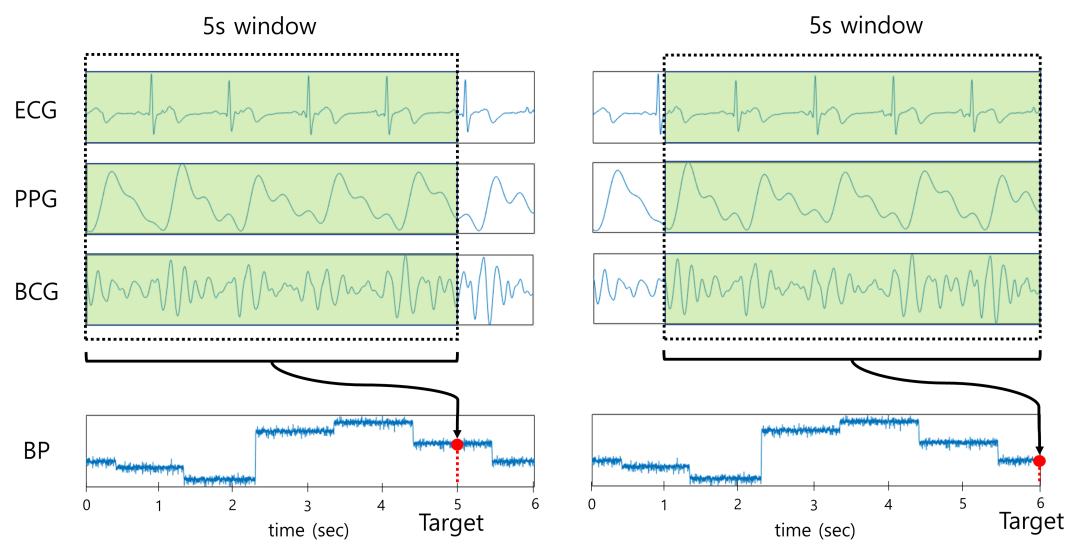

Figure 4. Data preprocessing of the deep learning model.

\subsection{Deep Learning Model}

\subsubsection{Convolutional Neural Network}

Convolutional neural networks (CNNs) have achieved considerable success in various challenging areas by extracting key features from a large amount of data such as images. Recently, CNNs have achieved remarkable performance in signal processing, especially in biomedical areas [24-28]. In this study, a CNN was used to extract key patterns automatically from the input signal.

\subsubsection{Bidirectional Gated Recurrent Unit}

RNNs have been applied for sequential modeling in natural language understanding and video processing. However, conventional RNNs have the problem of the gradient exploding or vanishing when the sequence is long [29]. To solve this long-term dependency problem, two representative RNN-based models have been proposed. One is the long short term memory (LSTM) developed by Hochreiter and Schmidhuber [30], and the other is the gated recurrent unit (GRU) introduced by Cho et al. [31]. Although both models have achieved much better performance than conventional RNN [32], the LSTM-based model requires more training time as it has more learning parameters than GRU. Therefore, the GRU layer was used in our model. The internal structure of GRU used in this study is illustrated in Figure 5.

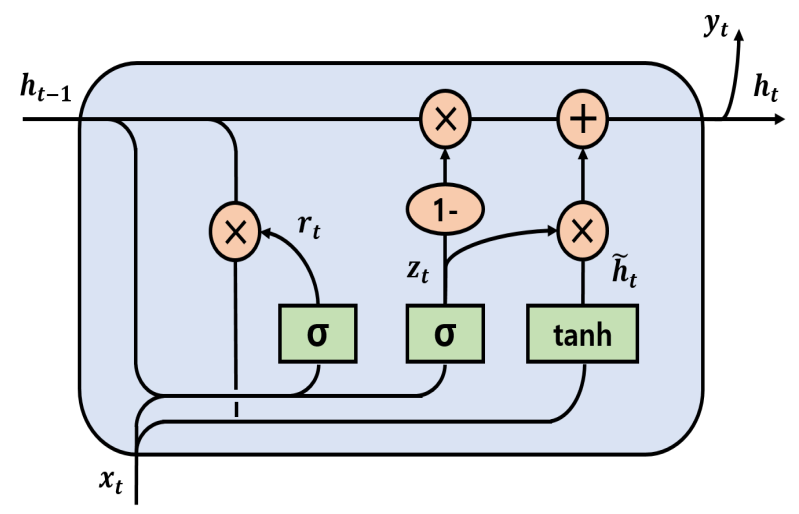

Figure 5. Internal structure of GRU.

There are two input vectors in a GRU cell at each time step, which include the previous hidden output vector $h_{t-1}$ and the current input vector $x_{t}$. The equation of the current hidden output vector $h_{t}$ can be expressed as follows: 


$$
\begin{array}{r}
z_{t}=\sigma\left(W_{z} \cdot\left[h_{t-1}, x_{t}\right]\right) \\
r_{t}=\sigma\left(W_{r} \cdot\left[h_{t-1}, x_{t}\right]\right) \\
\tilde{h}_{t}=\tanh \left(W_{h} \cdot\left[r_{t} \otimes h_{t-1}, x_{t}\right]\right) \\
h_{t}=\left(1-z_{t}\right) \otimes h_{t-1}+z_{t} \otimes \tilde{h}_{t}
\end{array}
$$

where $r_{t}$ and $z_{t}$ are the update gate and the reset gate vector, respectively. $W_{z}, W_{r}$, and $W_{h}$ are trainable weight parameters of each gate. $\tilde{h}_{t}$ is the candidate state that determines how much present information needs to be learned after the reset gate. $\sigma(\cdot)$ is a sigmoid function, and $\tanh (\cdot)$ is a hyperbolic tangential function. The symbol $\otimes$ denotes element-wise multiplication.

Conventional GRU is unidirectional, which means that each hidden state in a GRU cell only considers past information. Unlike unidirectional GRU, a bidirectional GRU (Bi-GRU) uses the information from both future and past feature vectors. Bi-GRU was used in this study instead of the conventional GRU. The $5 \mathrm{~s}$ segment was used as input in our model; hence, meaningful information existed in both directions at a specific time.

The Bi-GRU has two layers, forward and backward layers, as shown in Figure 6. The final output hidden vector $h_{t}$ can be represented as a concatenation of the forward hidden vector $\overrightarrow{h_{t}}$ and backward hidden vector $\overleftarrow{h_{t}}$, as shown:

$$
h_{t}=\overrightarrow{h_{t}} \oplus \overleftarrow{h_{t}}
$$

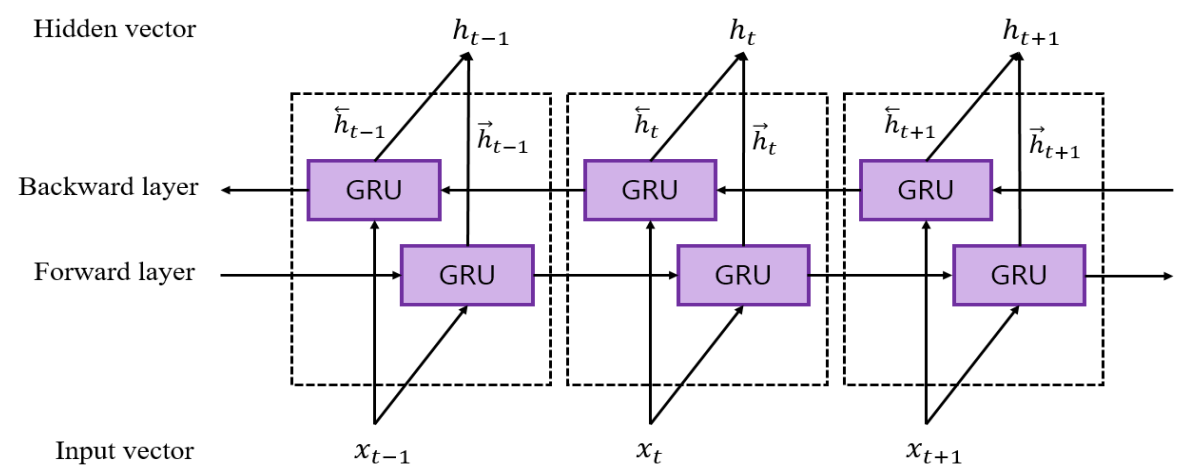

Figure 6. Bidirectional GRU structure.

\subsubsection{Attention Mechanism}

The main principle of the attention mechanism is that the model learns by focusing on the region of interest. The attention mechanism has recently been shown to be efficient in image captioning [33], neural machine translation [34,35], and signal processing [36]. Because sequential feature vectors from Bi-GRU in the proposed model may contribute differently for estimating BP values, we added an attention model to automatically train how much the feature vectors were important in each time step. Larger weights can be assigned to significant information using the attention mechanism, and various methods to apply the attention mechanism have been proposed [37]. In this work, the feed-forward attention model [38] was used, which is also known as the self-attention model. Given the Bi-GRU hidden state vector $\mathbf{h}_{\mathbf{i}}$ at every time step $i \in[1, N]$, the importance score $s_{i}$ was calculated through a score function $\operatorname{score}(\cdot)$ as follows:

$$
s_{i}=\operatorname{score}\left(\mathbf{W}_{\mathbf{s}} \mathbf{h}_{\mathbf{i}}+b\right)
$$

where Equation (4) can be represented as a single-layer perceptron having a trainable weight $\mathbf{W}_{\mathbf{s}}$ and a bias $b$. The score function can be set as an activation function in the neural network, and the tanh function was used in the proposed model. 
After obtaining each importance score $s_{i}$ for the hidden state vector $\mathbf{h}_{\mathbf{i}}$, the attention weight $a_{i}$ was evaluated using softmax function, expressed as:

$$
a_{i}=\operatorname{softmax}\left(s_{i}\right)=\frac{\exp \left(s_{i}\right)}{\sum_{i} \exp \left(s_{i}\right)}
$$

The final output vector $\mathbf{v}$ was obtained by calculating the weighted sum of the attention weight vector and the corresponding hidden state vector, as follows:

$$
\mathbf{v}=\sum_{i}^{N} a_{i} \mathbf{h}_{\mathbf{i}}
$$

\subsection{Proposed Model}

\subsubsection{Model Architecture}

Our proposed end-to-end deep learning network consists of a CNN layer, a Bi-GRU layer, and an attention layer, as shown in Figure 7. The deep learning models (including CNN and RNN) have shown promising performance in image-based time series recognition [39] and biosignal processing [25,40-42]. The attention mechanism has also been successfully applied to natural language processing [43].

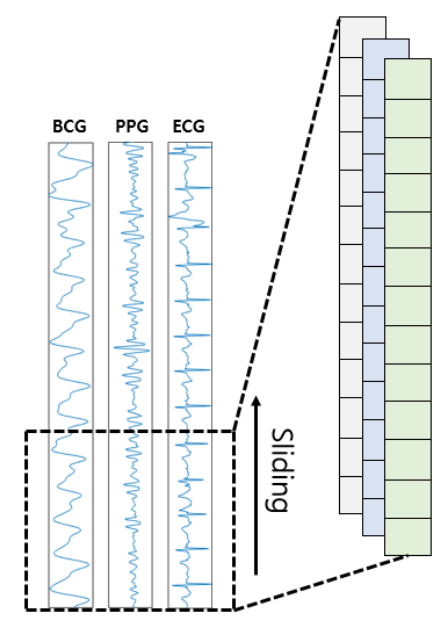

Input
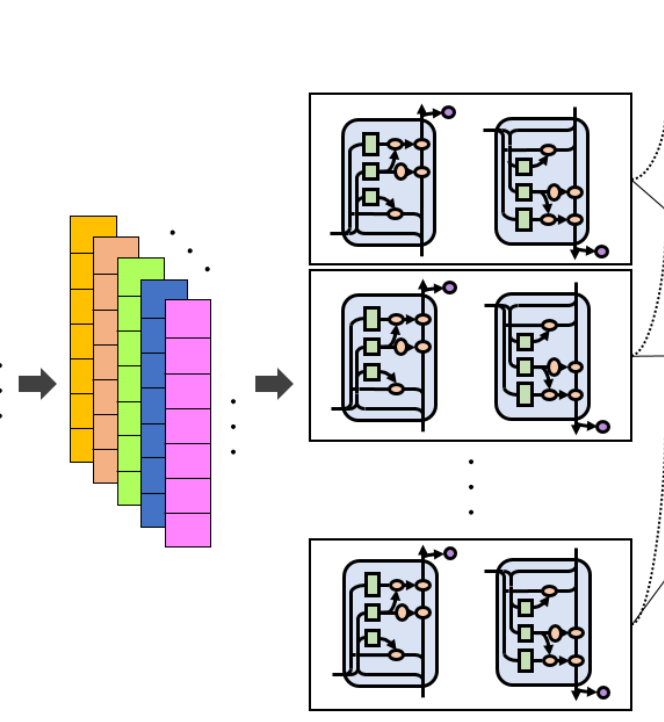

Bi-GRU

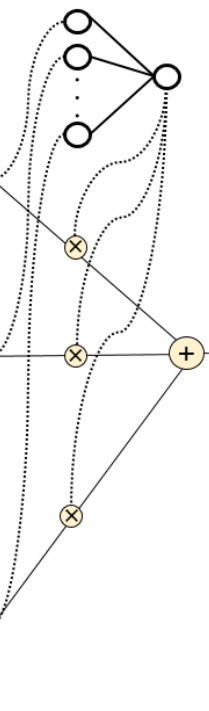

Attention

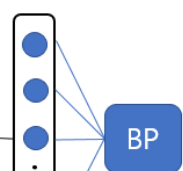

Output

Figure 7. Overall structure of the proposed network.

In our proposed model, the CNN structure shown in Figure 8 was designed by referring to the VGGNet structure [44]. A total of 10 convolution layers with a rectified linear unit (ReLU) activation function were used to extract spatial pattern vectors from signals. Each convolution layer was followed by a batch normalization layer to reduce the internal covariate shift [45]. The last layer of each convolution module was set to a max-pooling layer to reduce the length of the inputs. The same padding was applied to each convolution operation. Both kernel and pooling size were set to 3 , and the output channel size of the convolution layer was scaled up by a factor of 2 from 64 to 512 as it passed through each convolution module. 

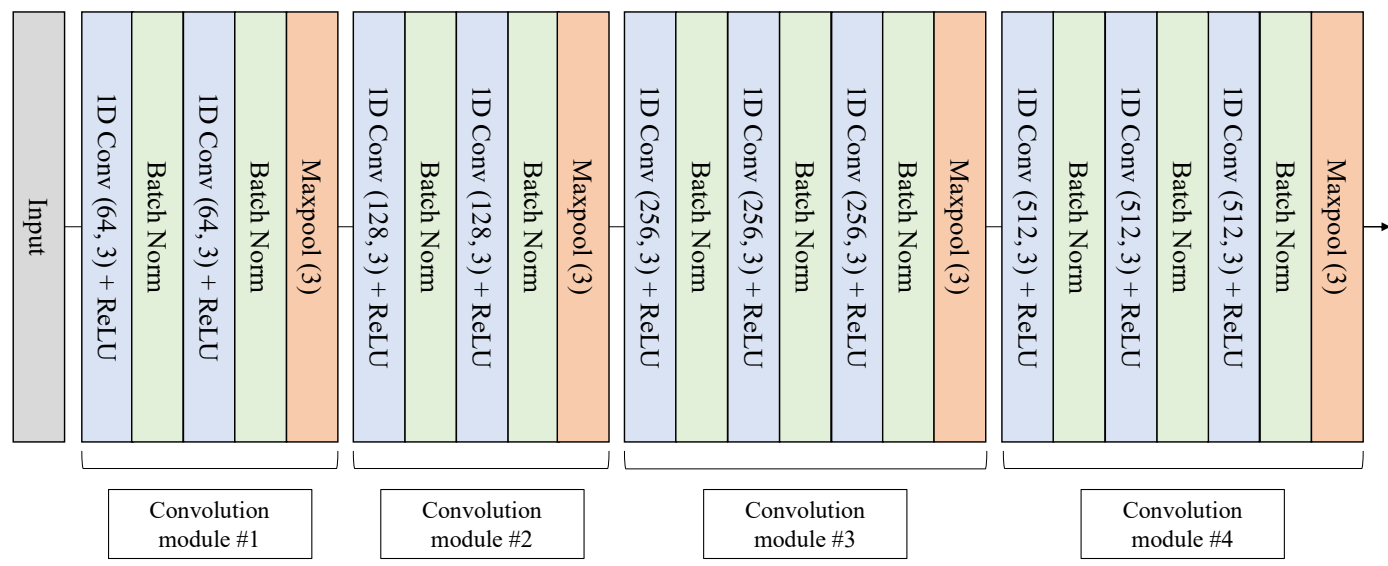

\section{Convolution} module \#4

Figure 8. CNN structure of the proposed model.

In the Bi-GRU phase, 64 hidden nodes were set up in each of the forward and backward layers, and 128 features were generated at each time step. The Bi-GRU layer can encode temporal information between features in our proposed model. In addition, representative features can be acquired in the Bi-GRU layer by reducing feature dimensions from 512 to 128 .

The output of the Bi-GRU hidden state vector is weighted by Equations (4)-(6), and, finally, these vectors are summed, and then the SBP and DBP values come out through 1-layer perceptron. The detailed structure of the proposed model is shown in Table 2.

Table 2. Detailed structure of the proposed model.

\begin{tabular}{|c|c|c|c|c|c|c|}
\hline Network & Layer & Shape & Out & Padding & Stride & Kerne \\
\hline \multirow{20}{*}{$\mathrm{CNN}$} & Conv & $625 \times 3$ & 64 & Same & 1 & 3 \\
\hline & \multicolumn{6}{|c|}{$\mathrm{BN}+\mathrm{ReLU}$} \\
\hline & Conv & $625 \times 64$ & 64 & Same & 1 & 3 \\
\hline & \multicolumn{6}{|c|}{$\mathrm{BN}+\mathrm{ReLU}$} \\
\hline & $\begin{array}{l}\text { Maxpool } \\
(\text { size = 3) }\end{array}$ & $625 \times 64$ & - & Same & 3 & - \\
\hline & Conv & $209 \times 64$ & 128 & Same & 1 & 3 \\
\hline & \multicolumn{6}{|c|}{$\mathrm{BN}+\mathrm{ReLU}$} \\
\hline & Conv & $209 \times 128$ & 128 & Same & 1 & 3 \\
\hline & \multicolumn{6}{|c|}{$\mathrm{BN}+\mathrm{ReLU}$} \\
\hline & $\begin{array}{l}\text { Maxpool } \\
(\text { size = 3) }\end{array}$ & $209 \times 128$ & - & Same & 3 & - \\
\hline & Conv & $70 \times 128$ & 256 & Same & 1 & 3 \\
\hline & \multicolumn{6}{|c|}{$\mathrm{BN}+\mathrm{ReLU}$} \\
\hline & Conv & $70 \times 256$ & 256 & Same & 1 & 3 \\
\hline & \multicolumn{6}{|c|}{$\mathrm{BN}+\mathrm{ReLU}$} \\
\hline & Conv & $70 \times 256$ & 256 & Same & 1 & 3 \\
\hline & \multicolumn{6}{|c|}{$\mathrm{BN}+\mathrm{ReLU}$} \\
\hline & $\begin{array}{l}\text { Maxpool } \\
(\text { size }=3)\end{array}$ & $70 \times 256$ & - & Same & 3 & - \\
\hline & Conv & $24 \times 256$ & 512 & Same & 1 & 3 \\
\hline & \multicolumn{6}{|c|}{$\mathrm{BN}+\mathrm{ReLU}$} \\
\hline & Conv & $24 \times 512$ & 512 & Same & 1 & 3 \\
\hline
\end{tabular}


Table 2. Cont.

\begin{tabular}{|c|c|c|c|c|c|c|}
\hline Network & Layer & Shape & Out & Padding & Stride & Kernel \\
\hline & \multicolumn{6}{|c|}{$\mathrm{BN}+\mathrm{ReLU}$} \\
\hline & Conv & $24 \times 512$ & 512 & Same & 1 & 3 \\
\hline & \multicolumn{6}{|c|}{$\mathrm{BN}+\mathrm{ReLU}$} \\
\hline & $\begin{array}{l}\text { Maxpool } \\
(\text { size = 3) }\end{array}$ & $24 \times 512$ & - & Same & 3 & - \\
\hline \multirow{3}{*}{ Bi-GRU } & Forward & $8 \times 512$ & 64 & & - & \\
\hline & Backward & $8 \times 512$ & 64 & & - & \\
\hline & \multicolumn{6}{|c|}{ Concatenation } \\
\hline \multirow{5}{*}{ Attention } & 1-layer perceptron & $8 \times 128$ & 1 & & - & \\
\hline & \multicolumn{6}{|c|}{ Activation tanh } \\
\hline & \multicolumn{6}{|c|}{ Softmax } \\
\hline & \multicolumn{6}{|c|}{ Weighted sum } \\
\hline & 1-layer perceptron & 128 & 2 & & - & \\
\hline
\end{tabular}

\subsubsection{Training Setting}

Seventy percent of the dataset was used for training, $10 \%$ for validation, and $20 \%$ for testing. The Adam optimizer [46] was used with a learning rate of $10^{-3}$ and decay of $10^{-4}$ to optimize the hyperparameters of the model. The learning rate was set to the optimal value empirically, and the initial weights were selected randomly. The mean squared error (MSE) was used for the loss function. The model was trained with the early stopping method with the patience of 10 in a maximum of 50 epochs, and the batch size was set to 512. As a computing environment for network training, the Keras deep learning framework with TensorFlow backend and NVIDIA GeForce RTX 2080Ti (NVIDIA corporation, Santa Clara, CA, USA) (with 11 GB VRAM) was used.

\section{Results}

In this section, three types of results are presented. First, the BP estimation results are compared for each combination of signals. Second, the performances of the models with and without attention are compared when all signals were used as inputs. Finally, the performance of our end-to-end deep learning model was compared with that of the multiple linear regression (MLR) model that used interval features from the characteristic point of each signal. The root-mean-square error (RMSE) and mean absolute error (MAE) were used as metrics of BP estimation accuracy. In addition, coefficient of determination $\left(R^{2}\right)$ values were calculated between the reference and estimated BP for all results. In addition, the Bland-Altman plot [47] was presented to increase the reliability of the results.

\subsection{Performance Comparison by Signal Combination}

A summary of the results from all combinations of the three signals is shown in Table 3. The model with ECG+PPG and ECG+PPG+BCG showed better performance for estimating BP than the other models. 
Table 3. Performance comparison for combinations of input signals without attention model and with attention. The $95 \%$ confidence interval is indicated below the error of the proposed model.

\begin{tabular}{|c|c|c|c|c|c|c|c|c|c|}
\hline \multirow[t]{2}{*}{ Model } & \multirow[t]{2}{*}{ Input } & \multicolumn{4}{|c|}{$\mathrm{SBP}(\mathrm{mmHg})$} & \multicolumn{4}{|c|}{ DBP (mmHg) } \\
\hline & & RMSE & MAE & SD & $\mathbf{R}^{2}$ & RMSE & MAE & SD & $\mathrm{R}^{2}$ \\
\hline \multirow{7}{*}{$\mathrm{CNN}+\mathrm{Bi}-\mathrm{GRU}$} & ECG & 7.02 & 5.51 & 4.66 & 0.24 & 5.16 & 4.06 & 3.45 & 0.27 \\
\hline & PPG & 6.88 & 5.34 & 4.60 & 0.28 & 5.73 & 4.45 & 4.09 & 0.14 \\
\hline & BCG & 7.24 & 5.59 & 5.03 & 0.20 & 5.29 & 4.06 & 3.71 & 0.22 \\
\hline & ECG, PPG & 5.83 & 4.46 & 4.06 & 0.46 & 4.74 & 3.70 & 3.37 & 0.38 \\
\hline & ECG, BCG & 6.74 & 5.30 & 4.60 & 0.31 & 4.82 & 3.74 & 3.27 & 0.34 \\
\hline & PPG, BCG & 6.44 & 4.86 & 4.50 & 0.36 & 5.04 & 3.88 & 3.62 & 0.27 \\
\hline & ECG, PPG, BCG & 5.87 & 4.51 & 4.14 & 0.48 & 4.73 & 3.71 & 3.39 & 0.40 \\
\hline $\begin{array}{c}\text { CNN+Bi-GRU } \\
\text { +Attention } \\
\text { (proposed model) }\end{array}$ & ECG, PPG, BCG & $\begin{array}{c}5.42 \\
{[1.97,8.87]}\end{array}$ & $\begin{array}{c}4.06 \\
{[1.53,6.59]}\end{array}$ & 4.04 & 0.52 & $\begin{array}{c}4.30 \\
{[0.94,7.72]}\end{array}$ & $\begin{array}{c}3.33 \\
{[0.61,6.05]}\end{array}$ & 3.42 & 0.49 \\
\hline
\end{tabular}

When a combination of multiple signals (ECG+PPG, ECG+BCG, PPG+BCG, and ECG+PPG+BCG) was used as input, the estimation accuracy was much better than the cases when a single signal (ECG, PPG, and BCG) was used as input. The detailed values are shown in Table 4.

Table 4. Mean values of RMSE, MAE, and $R^{2}$ when the input was a single signal and when it was a combination of multiple signals.

\begin{tabular}{ccccccc}
\hline Input & \multicolumn{3}{c}{ SBP (mmHg) } & \multicolumn{3}{c}{ DBP (mmHg) } \\
\hline & RMSE & MAE & mean $\mathbf{R}^{\mathbf{2}}$ & RMSE & MAE & mean $\mathbf{R}^{\mathbf{2}}$ \\
Single signal & 7.04 & 5.47 & 0.24 & 5.39 & 4.19 & 0.21 \\
Multiple signals & 6.21 & 4.78 & 0.40 & 4.83 & 3.76 & 0.35 \\
\hline
\end{tabular}

A repeated measurement analysis of variance (ANOVA) test was used to compare the performance when the combination of inputs was varied, and the differences between models were significant in both SBP and DBP estimation $(p<0.01)$. In addition, a paired $t$-test was performed between the results to compare each models. As shown in Tables 5 and 6 , the proposed model statistically outperformed the other methods regardless of the input in both SBP and DBP estimation.

Table 5. Results of paired $t$-test between various inputs for SBP estimation.

\begin{tabular}{ccccccccc}
\hline Inputs & ECG & PPG & BCG & ECG, PPG & ECG, BCG & BCG, PPG & ECG, BCG, PPG & Proposed Model \\
\hline ECG & & - & - & $p<0.05$ & - & - & $p<0.05$ & $p<0.05$ \\
PPG & & & - & $p<0.05$ & - & $p<0.05$ & $p<0.05$ & $p<0.05$ \\
BCG & & & & $p<0.05$ & - & $p<0.05$ & $p<0.05$ & $p<0.05$ \\
ECG, PPG & & & & & $p<0.05$ & - & - & $p<0.05$ \\
ECG, BCG & & & & & & - & $p<0.05$ & $p<0.05$ \\
BCG, PPG & & & & & & & - & $p<0.05$ \\
ECG, BCG, PPG & & & & & & $p<0.05$ \\
\hline
\end{tabular}

Table 6. Results of paired $t$-test between various inputs for DBP estimation.

\begin{tabular}{ccccccccc}
\hline Inputs & ECG & PPG & BCG & ECG, PPG & ECG, BCG & BCG, PPG & ECG, BCG, PPG & Proposed Model \\
\hline ECG & & - & - & - & $p<0.05$ & - & - & $p<0.05$ \\
PPG & & & - & $p<0.05$ & $p<0.05$ & $p<0.05$ & $p<0.05$ & $p<0.05$ \\
BCG & & & & - & - & - & - & $p<0.05$ \\
ECG, PPG & & & & & - & - & - & $p<0.05$ \\
ECG, BCG & & & & & & - & - & $p<0.05$ \\
BCG, PPG & & & & & & & & $p<0.05$ \\
ECG, BCG, PPG & & & & & & & $p<0.05$ \\
\hline
\end{tabular}

\subsection{Attention Mechanism Performance}

As shown in Table 3, the model with the attention mechanism showed lower RMSE and MAE values than the model without the attention mechanism. An example of BP estimation results is 
presented in Figure 9. The model with the attention mechanism estimated the fluctuation in BP more precisely than the model without the attention mechanism. The accuracy of the model can be improved with the attention mechanism by learning more intensively in the time step that contains relatively important information in the $5 \mathrm{~s}$ input data.

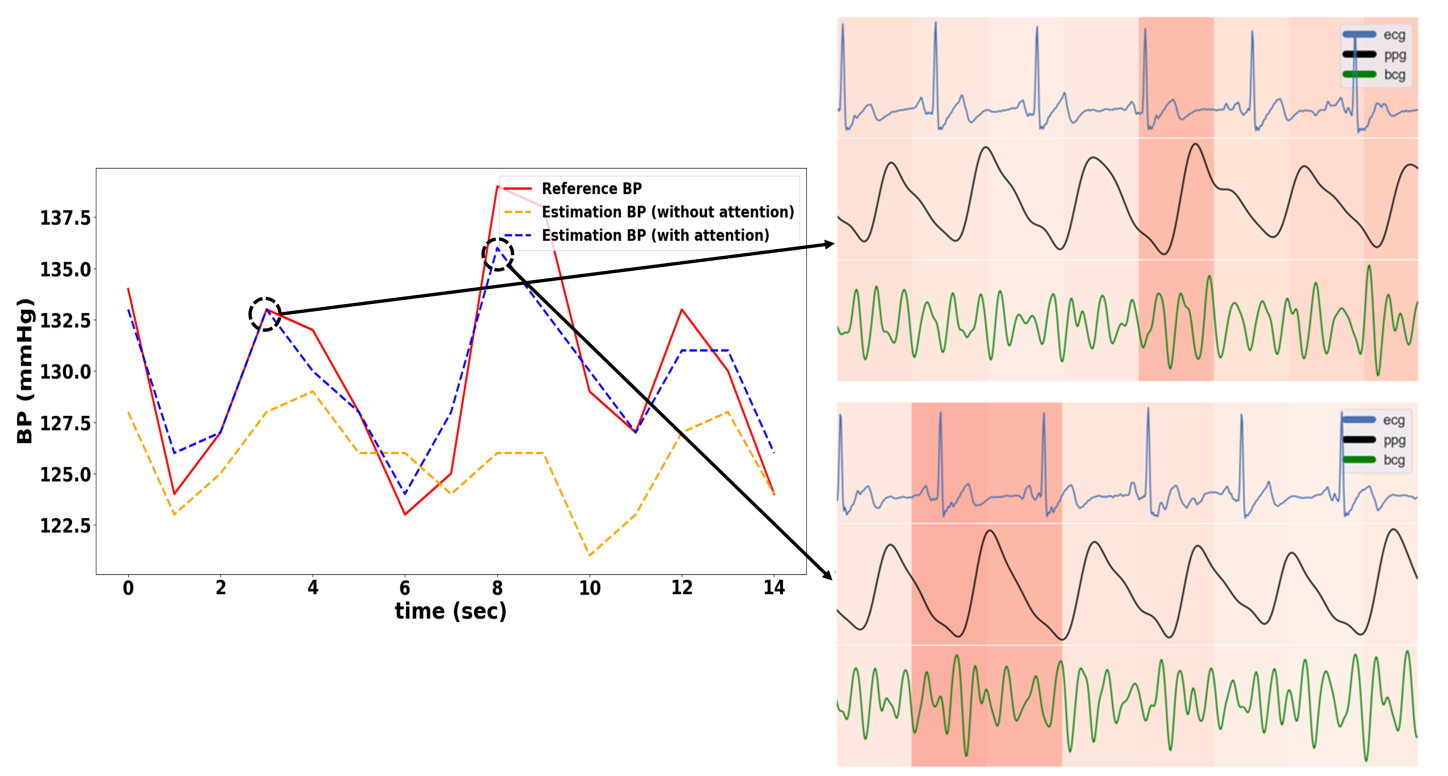

Figure 9. Left: Sample of the estimated BP with and without attention mechanism; Right: Heat map of the weights of the attention mechanism at the point where the error was low. The darker color denotes higher attention weight.

In addition, we found that the attention weight was high at a specific time by investigating the attention heat map. The process of generating the attention heat map is as follows: The length of the data was reduced from 625 (length resampled to $125 \mathrm{~Hz}$ ) to 8 by the CNN pooling layer, and thus Bi-GRU generated eight hidden state vectors. Accordingly, the original signal could be divided into eight sections, and the attention heat map was generated by assigning the attention weight obtained from the attention layer to each section.

The ANOVA test was conducted to interpret the attention weights of each timestep, and the results are summarized in Figure 10. The differences between timestep were statistically significant $(p<0.001)$, and the weights from timestep 2 to timestep 6 were significantly higher than other timesteps. The timestep 1 to timestep 7 includes $648 \mathrm{~ms}$ of feature information, and timestep 8 has $464 \mathrm{~ms}$ of feature information. Therefore, it means that the data from timestep 2 to timestep 6 (from $1.112 \mathrm{~s}$ to $3.704 \mathrm{~s}$ before the target BP) had more meaningful information to estimated BP. 


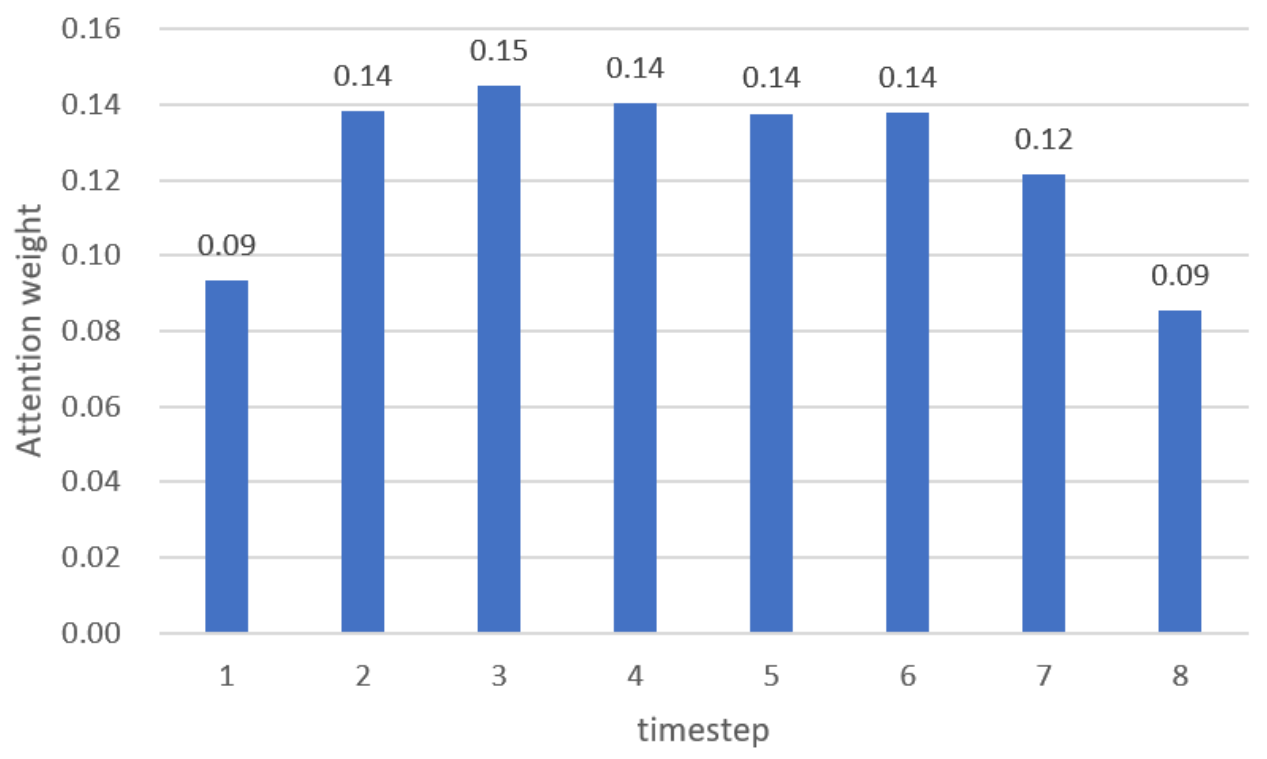

Figure 10. Mean attention weight across all datasets for each timestep.

Figure 11 shows the Bland-Altman plot between the estimated and reference BP. The limits of agreement (LOA) at 95\% confidence intervals for DBP and SBP were measured as $[-9.50,9.50]$ and $[-11.24,11.63]$, respectively. This means that $95 \%$ of the error was within [lower LOA, upper LOA]. Moreover, the mean error values between estimation and reference were 0.03 and 0.20 for DBP and SBP estimation, respectively, which means that the model had very little bias.
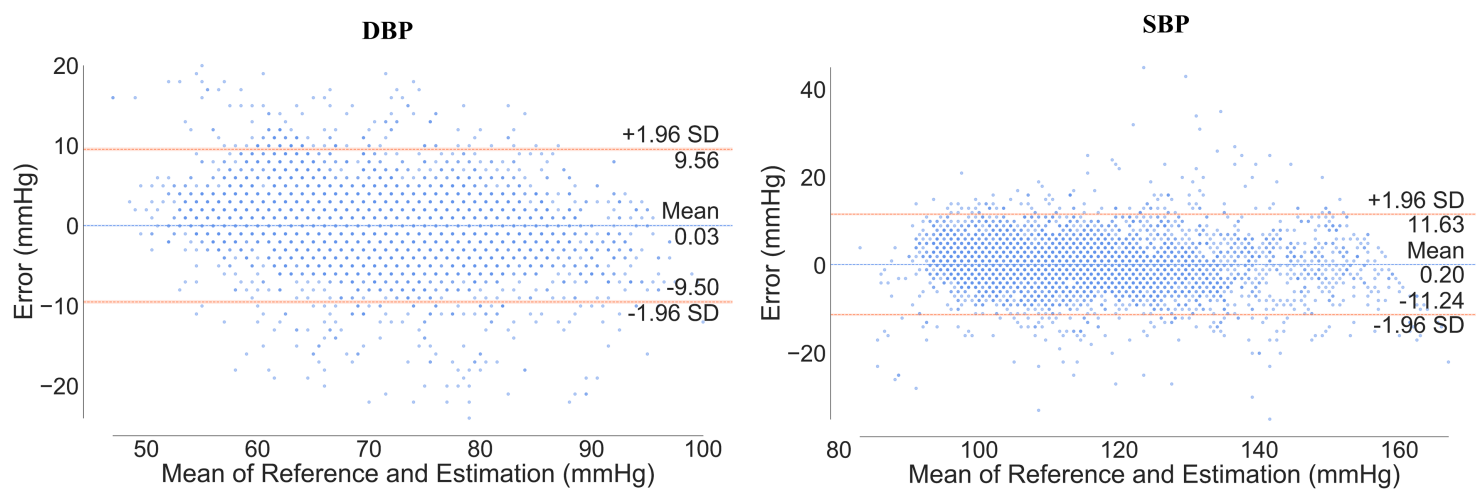

Figure 11. Bland-Altman plot of DBP and SBP. The orange line denotes the limit of agreement (LOA) and the blue line denotes the mean of difference error between reference and estimation.

Figure 12 shows the BP estimation result from two subjects. Overall, the estimated BP was similar to the reference BP, as shown in Figure 12a. However, as shown in Figure 12b, the error was high in cases where $\mathrm{BP}$ rapidly changes in a short time or if the overall range of $\mathrm{BP}$ is wide. 
a)

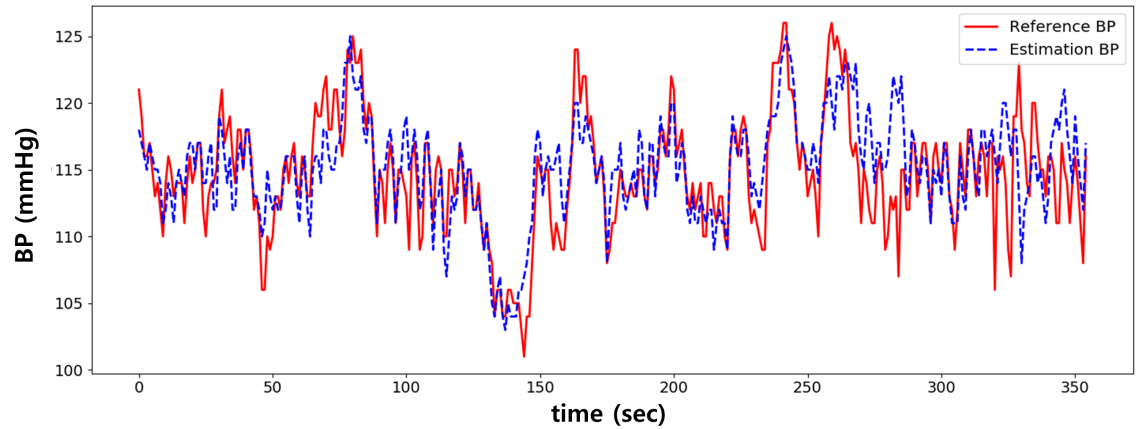

b)

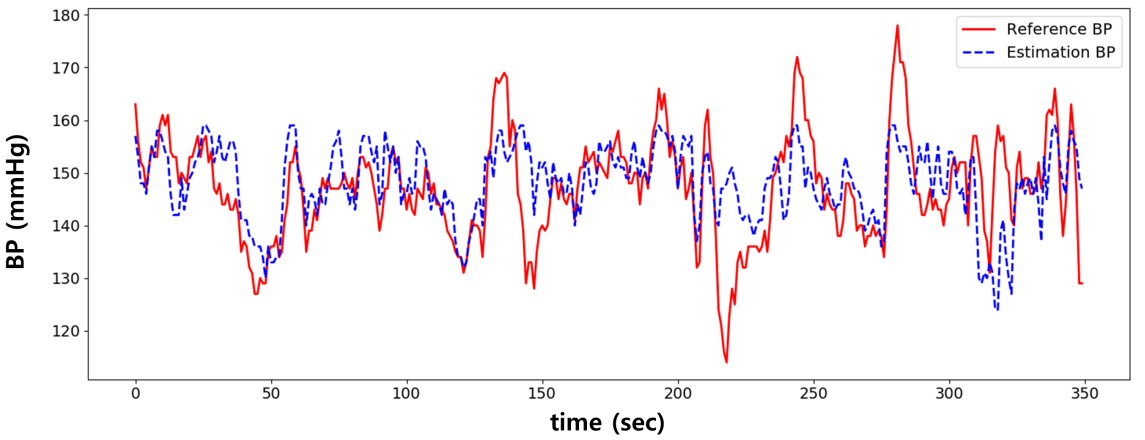

Figure 12. Comparison between estimated and reference BP. (a) is the best case; (b) is the worst case.

\subsection{Comparison to the Multiple Linear Regression Model}

The results of the proposed model were compared to the MLR model. The characteristic points from ECG, BCG, and PPG were detected to extract the features for the MLR model. ECG R-peak and the peak of the 1st derivative PPG were detected based on Pan and Tompkins's algorithm [48], and the BCG J peak was detected by finding the highest peak between $110 \mathrm{~ms}$ to $250 \mathrm{~ms}$ after each R-peak. False-positive peaks were excluded manually. Then, the R-R interval (RRI), PTT, and RJI were calculated from each cardiac cycle (Figure 13a). In addition, $21 \pm 11 \%$ of the cardiac cycles were excluded in cases where the peak was not detected because of motion artifacts (Figure 13b). The features were utilized as inputs of the MLR model, and SBP and DBP were used as dependent variables of the MLR model.

a)

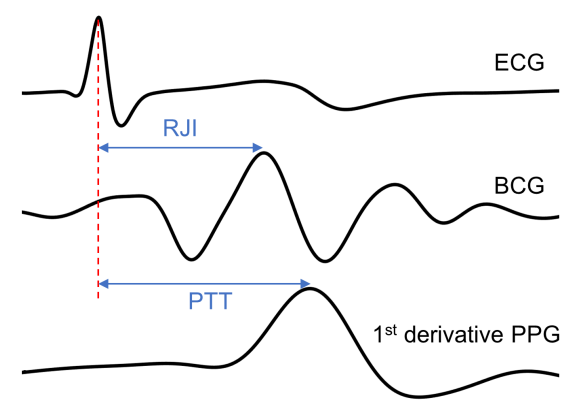

b)

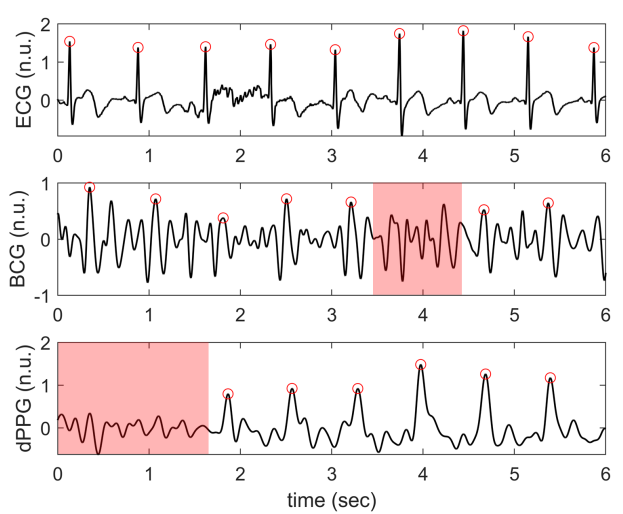

Figure 13. (a) example of the calculation of PTT and RJI in one cardiac cycle; (b) example of the excluded peaks. Red dots denote each characteristic point, and the red shaded region shows the area where peaks were not detected.

As summarized in Table 7, the MLR model showed lower performance for BP estimation than the proposed model. The input parameters of the MLR model were RRI, RJI, and PTT from ECG, PPG, and BCG, respectively. Although the input signal was the same in the MLR and proposed model, the MAE value of the MLR model was statistically higher in both SBP and DBP estimation $(p<0.05)$. 
Table 7. Comparison between proposed model and MLR model.

\begin{tabular}{ccccccccc}
\hline \multirow{2}{*}{ Model } & \multicolumn{3}{c}{ SBP (mmH) } & \multicolumn{4}{c}{ DBP (mmHg) } \\
& RMSE & MAE & SD & $\mathbf{R}^{2}$ & RMSE & MAE & SD & $\mathbf{R}^{\mathbf{2}}$ \\
\hline Proposed model & 5.42 & 4.06 & 4.04 & 0.52 & 4.30 & 3.33 & 3.42 & 0.49 \\
MLR & 6.40 & 5.19 & 3.45 & 0.26 & 4.75 & 3.85 & 2.69 & 0.22 \\
\hline
\end{tabular}

The scatter plots between PTT and SBP of the two cases are presented in Figure 14. Although the CC values between PTT and SBP were high in both cases, the $R^{2}$ value was 0.29 in the good case and only 0.05 in the bad case. The BP variation was not explained by the interval features alone, such as PTT, but our model could extract other features as well as the interval feature.

a)

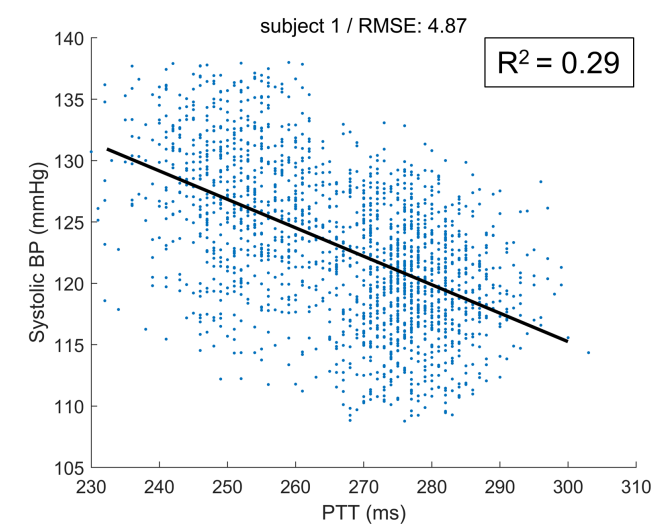

b)

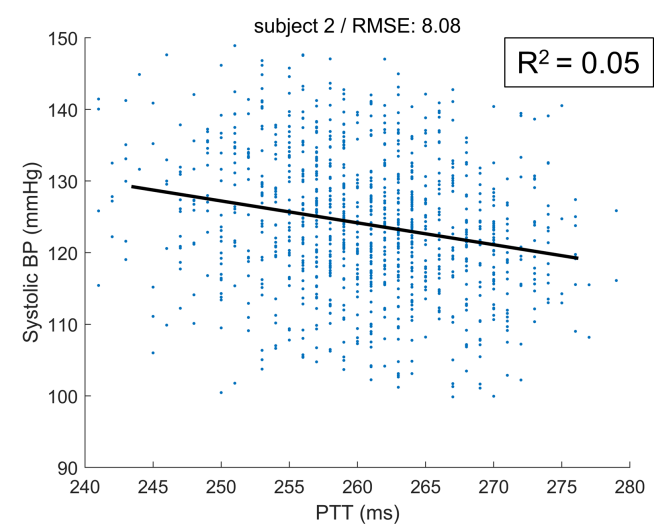

Figure 14. Scatter plots between PTT and Systolic BP of (a) good case and (b) bad case. The black line indicates a fitting line.

\section{Discussion}

\subsection{Main Contributions}

In this study, we proposed an end-to-end deep learning architecture that can estimate BP continuously using only raw signals without using feature engineering, which is time consuming and labor-intensive. In addition, we measured all the physiological signals such as ECG, PPG, and BCG mainly used in the previous BP estimation studies and compared the performances using all combinations of the signals as inputs to the model. Through these experiments, it was demonstrated that multiple signal involvement could improve the accuracy of the BP prediction over a single signal analysis. The proposed model achieved better performance than the MLR method using the conventional features, such as PTT and RJI. While the MLR model used features within only a bit corresponding to the BP to be estimated, the proposed model utilized multiple bits in $5 \mathrm{~s}$ length and learned the temporal relationship among the bits. Finally, we added the attention mechanism to improve the performance of the model. The results show that the data from previous bits as well as the bit right before the BP contributed to the estimation of the BP.

\subsection{Result Interpretation from Global Standard Perspective of BP Monitoring}

There are two types of BP monitoring global standards used as a performance indicators in most studies. One is the Association for the Advancement of the Medical Instrumentation (AAMI) standard [49], and the other is the British Hypertension Society (BHS) standard [50]. Tables 8 and 9 show the results of using the proposed model against these standards. Though the study population of this study was 15 , which was insufficient because the AAMI and BHS standards require at least 85 subjects, the results complied with the AAMI and BHS standards in grade A for both SBP and DBP. 
Table 8. Performance comparison with the AAMI standard.

\begin{tabular}{cccc}
\hline & & Mean Error & Standard Deviation \\
\hline \multirow{2}{*}{ AAMI standard } & SBP, DBP & $\leq \mathbf{5 m H g})$ & $\leq \mathbf{8}(\mathrm{mmHg})$ \\
\hline \multirow{2}{*}{ Proposed model } & SBP & -0.20 & 5.83 \\
& DBP & -0.02 & 4.91 \\
\hline
\end{tabular}

Table 9. Performance comparison with the BHS standard.

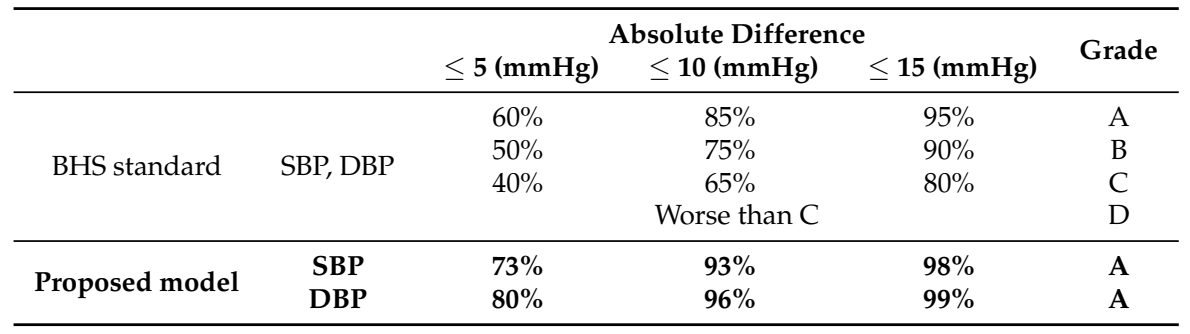

\subsection{Comparison Result With Related Works}

The performances of the models used in related studies are shown in Table 10. Chan et al. proposed a linear regression model based on PTT [14]. Even though the study used mean error, the error was high and the data size was unspecified. Kachuee et al. [15], Kurylyak et al. [17], Su et al. [16], and Wang et al. [18] also proposed feature-based approaches. The studies have suggested various methods to extract the features, which need a lot of time. On the other hand, our proposed model used only a bandpass filtering and did not require a complicated feature extraction process.

Lee et al. proposed a BP estimation model using only two channels of BCG [13]. Though estimating BP using only BCG was meaningful, the evaluation was conducted with the one time BP measurement, not a continuous BP measurement. Tanveer et al. achieved excellent performance using raw signals from ECG and PPG [20], but the window length to estimate BP was very long, 16 s, while we used $5 \mathrm{~s}$ input. In addition, the authors noted that the performance of their proposed model is highly dependent on the proper division of ECG and PPG cycles. It means that additional work such as peak detection is required, and the quality of the signal affects the performance of model. Slapnivcar et al. also proposed a deep learning model using the PPG raw signal [19], but its performance was low compared to other studies. In addition, it is difficult to evaluate the model accurately because other evaluation metrics were not presented. 
Table 10. Performance comparison with related works.

\begin{tabular}{|c|c|c|c|c|c|c|c|}
\hline \multirow{2}{*}{ Author } & \multirow{2}{*}{ Data Size } & \multirow{2}{*}{ Calibration } & \multirow{2}{*}{ Model } & \multicolumn{2}{|c|}{ Input } & \multirow{2}{*}{$\frac{\mathrm{SBP}(\mathrm{mmHg})}{\text { Error }}$} & \multirow{2}{*}{$\frac{\text { DBP }(\mathrm{mmHg})}{\text { Error }}$} \\
\hline & & & & Inputs & Signal & & \\
\hline Chan et al. [14] & Unspecified & Cal-based & $\begin{array}{l}\text { Linear } \\
\text { regression }\end{array}$ & $\begin{array}{c}\text { Feature } \\
\text { (PTT) }\end{array}$ & $\begin{array}{l}\text { ECG } \\
\text { PPG }\end{array}$ & $\begin{array}{l}\text { ME : } 7.49 \\
\text { STD : } 8.82\end{array}$ & $\begin{array}{c}\text { ME : } 4.08 \\
\text { STD : } 5.62\end{array}$ \\
\hline \multirow{2}{*}{$\begin{array}{l}\text { Kachuee } \\
\text { et al. [15] }\end{array}$} & \multirow{2}{*}{$\begin{array}{l}1000 \text { subjects } \\
10 \text { min } \\
\text { (MIMIC } 3 \text { ) }\end{array}$} & Cal-based & \multirow{2}{*}{ AdaBoost } & \multirow{2}{*}{ Features } & \multirow{2}{*}{$\begin{array}{l}\text { ECG } \\
\text { PPG }\end{array}$} & $\begin{array}{l}\text { MAE : } 8.21 \\
\text { STD : } 5.45\end{array}$ & $\begin{array}{l}\text { MAE : } 4.31 \\
\text { STD : } 3.52\end{array}$ \\
\hline & & Cal-free & & & & $\begin{array}{l}\text { MAE : } 11.17 \\
\text { STD : } 10.09\end{array}$ & $\begin{array}{l}\text { MAE : } 5.35 \\
\text { STD : } 6.14\end{array}$ \\
\hline $\begin{array}{l}\text { Kurylyak } \\
\text { et al. [17] }\end{array}$ & $\begin{array}{c}15,000 \\
\text { heartbeats }\end{array}$ & Cal-based & $\begin{array}{c}\text { Deep } \\
\text { learning } \\
\text { (ANN) }\end{array}$ & Features & PPG & $\begin{array}{l}\text { ME : } 3.80 \\
\text { STD : } 3.46\end{array}$ & $\begin{array}{c}\text { ME : } 2.21 \\
\text { STD : } 2.09\end{array}$ \\
\hline Lee et al. [13] & 30 subjects & Cal-based & $\begin{array}{l}\text { Deep } \\
\text { learning } \\
\text { (ANN) }\end{array}$ & $\begin{array}{l}\text { Feature } \\
\text { (IPD) }\end{array}$ & BCG & $\begin{array}{c}\text { ME : } 0.01 \\
\text { STD : } 6.75\end{array}$ & $\begin{array}{l}\text { ME : } 0.05 \\
\text { STD : } 5.83\end{array}$ \\
\hline \multirow{2}{*}{$\begin{array}{c}\text { Slapnivcar } \\
\text { et al. [19] }\end{array}$} & \multirow{2}{*}{$\begin{array}{l}510 \text { subjects } \\
700 \mathrm{~h} \\
\text { (MIMIC 3) }\end{array}$} & Cal-based & \multirow{2}{*}{$\begin{array}{c}\text { Deep } \\
\text { learning } \\
\text { (ResNet) }\end{array}$} & \multirow{2}{*}{ Raw } & \multirow{2}{*}{ PPG } & MAE : 9.43 & MAE : 6.88 \\
\hline & & Cal-free & & & & MAE : 15.41 & MAE : 12.38 \\
\hline Su et al. [16] & $\begin{array}{l}84 \text { subjects } \\
10 \mathrm{~min}\end{array}$ & Cal-based & $\begin{array}{c}\text { Deep } \\
\text { learning } \\
(\mathrm{RNN})\end{array}$ & Features & $\begin{array}{l}\text { ECG } \\
\text { PPG }\end{array}$ & RMSE : 3.73 & RMSE : 2.43 \\
\hline $\begin{array}{l}\text { Tanveer } \\
\text { et al. [20] }\end{array}$ & $\begin{array}{l}39 \text { subjects } \\
\text { (MIMIC 1) }\end{array}$ & Cal-based & $\begin{array}{c}\text { Deep } \\
\text { learning } \\
\text { (ANN+ } \\
\text { LSTM) }\end{array}$ & Raw & $\begin{array}{l}\text { ECG } \\
\text { PPG }\end{array}$ & $\begin{array}{l}\text { RMSE : } 1.27 \\
\text { MAE : } 0.93\end{array}$ & $\begin{array}{l}\text { RMSE : } 0.73 \\
\text { MAE : } 0.52\end{array}$ \\
\hline Wang et al. [18] & $\begin{array}{c}58,795 \\
\text { intervals } \\
\text { of PPG } \\
\text { (MIMIC 1) }\end{array}$ & Cal-based & $\begin{array}{c}\text { Deep } \\
\text { learning } \\
(\mathrm{ANN})\end{array}$ & Features & PPG & $\begin{array}{c}\text { MAE : } 4.02 \\
\text { STD : } 2.79\end{array}$ & $\begin{array}{l}\text { MAE : } 2.27 \\
\text { STD : } 1.82\end{array}$ \\
\hline \multirow{3}{*}{ This study } & \multirow{3}{*}{$\begin{array}{l}15 \text { subjects } \\
30 \mathrm{~min}\end{array}$} & \multirow{3}{*}{ Cal-based } & \multirow{2}{*}{$\begin{array}{c}\text { Deep } \\
\text { learning } \\
\text { (CNN+ } \\
\text { Bi-GRU) }\end{array}$} & \multirow{3}{*}{ Raw } & BCG & $\begin{array}{l}\text { ME : }-0.82 \\
\text { STD : } 7.50\end{array}$ & $\begin{array}{l}\text { ME : }-0.97 \\
\text { STD : } 5.36\end{array}$ \\
\hline & & & & & $\begin{array}{l}\text { ECG } \\
\text { PPG }\end{array}$ & $\begin{array}{l}\text { MAE : } 4.46 \\
\text { STD : } 4.06\end{array}$ & $\begin{array}{l}\text { MAE }: 3.70 \\
\text { STD }: 3.37\end{array}$ \\
\hline & & & $\begin{array}{c}\text { Deep } \\
\text { learning } \\
(\mathrm{CNN}+ \\
\text { Bi-GRU+ } \\
\text { Attention) }\end{array}$ & & $\begin{array}{l}\text { ECG } \\
\text { PPG } \\
\text { BCG }\end{array}$ & $\begin{array}{l}\text { MAE : } 4.06 \\
\text { STD : } 4.04\end{array}$ & $\begin{array}{l}\text { MAE : } 3.33 \\
\text { STD : } 3.42\end{array}$ \\
\hline
\end{tabular}

\subsection{Limitations of the Study}

Kauchee et al. [15] and Slapnivcar et al. [19] proposed a calibration-free (cal-free) model that did not require a calibration of each subject. In this study, we focused on a calibration-based (cal-based) model, but designing a cal-free model that is not affected by subject characteristics is another important issue. Thus, we also attempted the development of a cal-free method using leave-one-subject-out (LOSO) method, and the results were summarized in Table 11. The performance of the cal-free model was inferior to that of a cal-based model. In addition, the result did not comply with the global standard mentioned in Section 4.2 and needs to be improved in further study.

Table 11. Performance comparison between calibration-free and calibration-based methods using the proposed model.

\begin{tabular}{cccccccccc}
\hline Input & Method & \multicolumn{3}{c}{ SBP (mmHg) } & \multicolumn{4}{c}{ DBP (mmHg) } \\
\hline & & RMSE & MAE & SD & $\mathbf{R}^{2}$ & RMSE & MAE & SD & $\mathbf{R}^{2}$ \\
ECG, PPG, BCG & Cal-based & 5.42 & 4.06 & 4.04 & 0.52 & 4.3 & 3.33 & 3.42 & 0.49 \\
& Cal-free & 13.14 & 9.70 & 8.86 & 0.23 & 7.55 & 5.79 & 4.84 & 0.44 \\
\hline
\end{tabular}


In this study, data were measured in a short time of $30 \mathrm{~min}$, hence the performance of the method while predicting long-term BP should be considered. In addition, as mentioned in Section 4.2, the number of subjects (global standard: minimum of 85 subjects) was not sufficient; however, we used inputs with $5 \mathrm{~s}$ lengths and total sample size was 26,600,014, which was enough to evaluate the model. In addition, the data of patients with hypertension were not included in the study because most of the subjects were healthy 20 -year-olds with no diseases. However, the $95 \%$ confidence interval of the BP that we used in the experiment was [86.34, 143.74] and [51.28, 88.74] for SBP and DBP, respectively. Moreover, about $9 \%$ and $5 \%$ of the BP data were in hypertension stage 1 and stage 2 ranges [51], though no subject was diagnosed as a hypertension patient.

\section{Conclusions and Future Work}

In this paper, we proposed an end-to-end deep learning algorithm to estimate BP with attention mechanism. The proposed method was designed to improve the performance of estimating BP and to overcome the limitations of previous study. The method does not require a feature extraction process, which is a costly and laborious task, as ECG, PPG, and BCG signals were used as inputs after a simple bandpass filter was applied. In addition, the method enables a noninvasive and continuous BP monitoring as the physiological signals could be measured non-invasively. As mentioned in Section 3, the model with the attention mechanism outperformed others. These results imply that other physiological signal processing methods can be improved with the attention mechanism. In the future study, we will analyze which patterns of signals were weighted by the attention mechanism. In addition, we will consider a sufficiently large number of subjects and long-term data to optimize the model and improve performance.

Author Contributions: Conceptualization and methodology, D.L. and H.E.; validation and software, H.E.; formal analysis, D.L., H.E., and I.S.; investigation, D.L. and Y.L.; resources, D.L.; data curation, D.L., H.E., and S.H.; writing-original draft preparation, D.L. and H.E.; writing—review and editing, K.P. and C.P.; visualization, H.E. and Y.S.H.; supervision, C.P.; project administration, K.P. All authors have read and agreed to the published version of the manuscript.

Funding: This work was supported by the National Research Foundation of Korea (NRF) grant funded by the Korea government (MSIT) (NRF-2017R1A5A1015596), and the Research Grant of Kwangwoon University in 2020.

Conflicts of Interest: The authors declare no conflict of interest. The funders had no role in the design of the study; in the collection, analyses, or interpretation of data; in the writing of the manuscript, or in the decision to publish the results.

\section{Abbreviations}

The following abbreviations are used in this manuscript:

$\begin{array}{ll}\text { BP } & \text { Blood pressure } \\ \text { SBP } & \text { Systolic blood pressure } \\ \text { DBP } & \text { Diastolic blood pressure } \\ \text { ABP } & \text { Arterial blood pressure } \\ \text { ICU } & \text { Intensive care unit } \\ \text { PWV } & \text { Pulse wave velocity } \\ \text { PTT } & \text { Pulse transit time } \\ \text { ECG } & \text { Electrocardiogram } \\ \text { PPG } & \text { Photoplethysmogram } \\ \text { BCG } & \text { Ballistocardiogram } \\ \text { PVDF } & \text { Polyvinylidene fluoride } \\ \text { RJI } & \text { R-J interval } \\ \text { ANN } & \text { Artificial neural network } \\ \text { MLR } & \text { Multiple linear regression } \\ \text { RRI } & \text { R-R interval } \\ \text { CNN } & \text { Convolutional neural network } \\ \text { Bi-GRU } & \text { Bidirectional gated unit } \\ \text { ReLU } & \text { Rectified linear unit } \\ \text { RNN } & \text { Recurrent neural network }\end{array}$




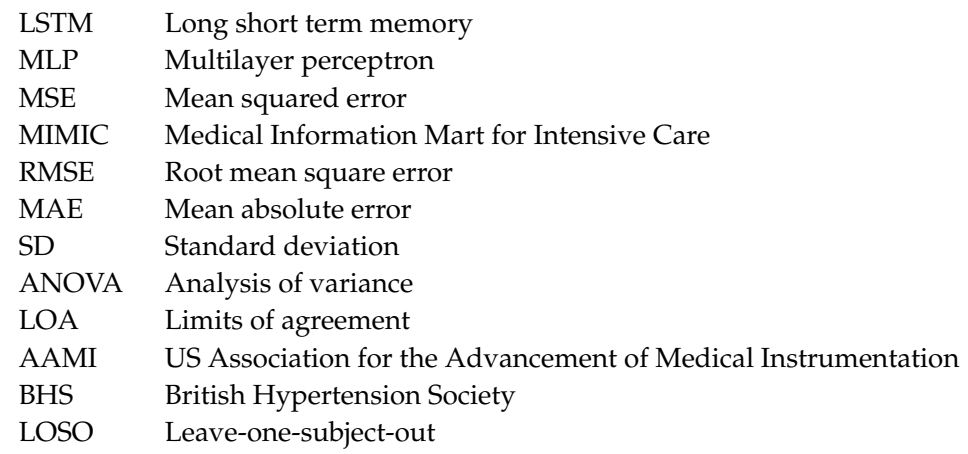

\section{References}

1. World Health Organization (WHO), Hypertension. Available online: https://www.who.int/news-room/ fact-sheets/detail/hypertension (accessed on 18 April 2020).

2. Ogedegbe, G.; Pickering, T. Principles and techniques of blood pressure measurement. Cardiol. Clin. 2010, 28, 571-586. [CrossRef] [PubMed]

3. Yoo, S.; Baek, H.; Doh, K.; Jeong, J.; Ahn, S.; Oh, I.Y.; Kim, K. Validation of the mobile wireless digital automatic blood pressure monitor using the cuff pressure oscillometric method, for clinical use and self-management, according to international protocols. Open Biomed. Eng. Lett. 2018, 8, 399-404. [CrossRef] [PubMed]

4. Wu, D.; Xu, L.; Abbott, D.; Hau, W.K.; Ren, L.; Zhang, H.; Wong, K.K. Analysis of beat-to-beat blood pressure variability response to the cold pressor test in the offspring of hypertensive and normotensive parents. Hypertens. Res. 2017, 40, 581-589. [CrossRef] [PubMed]

5. Ding, X.; Zhang, Y.T. Pulse transit time technique for cuffless unobtrusive blood pressure measurement: from theory to algorithm. Biomed. Eng. Lett. 2019, 9, 37-52. [CrossRef] [PubMed]

6. Wong, M.Y.M.; Poon, C.C.Y.; Zhang, Y.T. An evaluation of the cuffless blood pressure estimation based on pulse transit time technique: a half year study on normotensive subjects. Cardiovasc. Eng. 2009, 9, 32-38. [CrossRef]

7. Chen, W.; Kobayashi, T.; Ichikawa, S.; Takeuchi, Y.; Togawa, T. Continuous estimation of systolic blood pressure using the pulse arrival time and intermittent calibration. Med. Biol. Eng. Comput. 2000, 38, 569-574. [CrossRef]

8. Poon, C.; Zhang, Y. Cuff-less and noninvasive measurements of arterial blood pressure by pulse transit time. In Proceedings of the 2005 IEEE Engineering in Medicine and Biology 27th Annual Conference, Shanghai, China, 17-18 January 2006; pp. 5877-5880.

9. Baek, H.J.; Chung, G.S.; Kim, K.K.; Park, K.S. A smart health monitoring chair for nonintrusive measurement of biological signals. IEEE Trans. Inf. Technol. Biomed. 2011, 16, 150-158. [CrossRef]

10. Ding, X.R.; Zhang, Y.T.; Liu, J.; Dai, W.X.; Tsang, H.K. Continuous cuffless blood pressure estimation using pulse transit time and photoplethysmogram intensity ratio. IEEE Trans. Biomed. Eng. 2016, 63, 964-972. [CrossRef]

11. Kim, C.S.; Carek, A.M.; Mukkamala, R.; Inan, O.T.; Hahn, J.O. Ballistocardiogram as proximal timing reference for pulse transit time measurement: Potential for cuffless blood pressure monitoring. IEEE Trans. Biomed. Eng. 2015, 62, 2657-2664. [CrossRef]

12. Shin, J.H.; Lee, K.M.; Park, K.S. Non-constrained monitoring of systolic blood pressure on a weighing scale. Physiol. Meas. 2009, 30, 679. [CrossRef]

13. Lee, K.J.; Roh, J.; Cho, D.; Hyeong, J.; Kim, S. A Chair-Based Unconstrained/Nonintrusive Cuffless Blood Pressure Monitoring System Using a Two-Channel Ballistocardiogram. Sensors 2019, 19, 595. [CrossRef]

14. Chan, K.; Hung, K.; Zhang, Y. Noninvasive and cuffless measurements of blood pressure for telemedicine. In Proceedings of the 23rd Annual International Conference of the IEEE Engineering in Medicine and Biology Society, Istanbul, Turkey, 25-28 October 2001; pp. 3592-3593.

15. Kachuee, M.; Kiani, M.M.; Mohammadzade, H.; Shabany, M. Cuffless blood pressure estimation algorithms for continuous health-care monitoring. IEEE Trans. Biomed. Eng. 2016, 64, 859-869. [CrossRef] [PubMed] 
16. Su, P.; Ding, X.R.; Zhang, Y.T.; Liu, J.; Miao, F.; Zhao, N. Long-term blood pressure prediction with deep recurrent neural networks. In Proceedings of the 2018 IEEE EMBS International Conference on Biomedical \& Health Informatics (BHI), Las Vegas, NV, USA, 4-7 March 2018; pp. 323-328.

17. Kurylyak, Y.; Lamonaca, F.; Grimaldi, D. A Neural Network-based method for continuous blood pressure estimation from a PPG signal. In Proceedings of the 2013 IEEE International Instrumentation and Measurement Technology Conference (I2MTC), Minneapolis, MN, USA, 6-9 May 2013; pp. 280-283.

18. Wang, L.; Zhou, W.; Xing, Y.; Zhou, X. A novel neural network model for blood pressure estimation using photoplethesmography without electrocardiogram. J. Healthcare Eng. 2018, 2018. [CrossRef] [PubMed]

19. Slapničar, G.; Mlakar, N.; Luštrek, M. Blood Pressure Estimation from Photoplethysmogram Using a Spectro-Temporal Deep Neural Network. Sensors 2019, 19, 3420. [CrossRef]

20. Tanveer, M.S.; Hasan, M.K. Cuffless blood pressure estimation from electrocardiogram and photoplethysmogram using waveform based ANN-LSTM network. Biomed. Signal Process. Control 2019, 51, 382-392. [CrossRef]

21. Physiolab, Busan, Korea. Available online: http:/ / www.physiolab.co.kr (accessed on 18 April 2020).

22. Goldberger, A.L.; Amaral, L.A.; Glass, L.; Hausdorff, J.M.; Ivanov, P.C.; Mark, R.G.; Mietus, J.E.; Moody, G.B.; Peng, C.K.; Stanley, H.E.; et al. PhysioBank, PhysioToolkit, and PhysioNet: Components of a new research resource for complex physiologic signals. Circulation 2000, 101, e215-e220. [CrossRef]

23. Wen, X.; Huang, Y.; Wu, X.M.; Zhang, B. A Feasible Feature Extraction Method for Atrial Fibrillation Detection from BCG. IEEE J. Biomed. Health Inf. 2019, 24, 1093-1103. [CrossRef]

24. Rundo, F.; Conoci, S.; Ortis, A.; Battiato, S. An advanced bio-inspired photoplethysmography (PPG) and ECG pattern recognition system for medical assessment. Sensors 2018, 18, 405. [CrossRef]

25. Yıldırım, Ö.; Pławiak, P.; Tan, R.S.; Acharya, U.R. Arrhythmia detection using deep convolutional neural network with long duration ECG signals. Comput. Biol. Med. 2018, 102, 411-420. [CrossRef]

26. Ullah, I.; Hussain, M.; Aboalsamh, H. An automated system for epilepsy detection using EEG brain signals based on deep learning approach. Expert Syst. Appl. 2018, 107, 61-71. [CrossRef]

27. Dey, D.; Chaudhuri, S.; Munshi, S. Obstructive sleep apnoea detection using convolutional neural network based deep learning framework. Biomed. Eng. Lett. 2018, 8, 95-100. [CrossRef] [PubMed]

28. Khagi, B.; Lee, B.; Pyun, J.Y.; Kwon, G.R. CNN Models Performance Analysis on MRI images of OASIS dataset for distinction between Healthy and Alzheimer's patient. In Proceedings of the 2019 International Conference on Electronics, Information, and Communication (ICEIC), Auckland, New Zealand, 22-25 January 2019; pp. 1-4.

29. Bengio, Y.; Simard, P.; Frasconi, P. Learning long-term dependencies with gradient descent is difficult. IEEE Trans. Neural Networks 1994, 5, 157-166. [CrossRef] [PubMed]

30. Hochreiter, S.; Schmidhuber, J. Long short-term memory. Neural Comput. 1997, 9, 1735-1780. [CrossRef] [PubMed]

31. Cho, K.; Van Merriënboer, B.; Gulcehre, C.; Bahdanau, D.; Bougares, F.; Schwenk, H.; Bengio, Y. Learning phrase representations using RNN encoder-decoder for statistical machine translation. arXiv 2014, arXiv:1406.1078.

32. Chung, J.; Gulcehre, C.; Cho, K.; Bengio, Y. Empirical evaluation of gated recurrent neural networks on sequence modeling. arXiv 2014, arXiv:1412.3555.

33. Xu, K.; Ba, J.; Kiros, R.; Cho, K.; Courville, A.; Salakhudinov, R.; Zemel, R.; Bengio, Y. Show, attend and tell: Neural image caption generation with visual attention. In Proceedings of the International Conference on Machine Learning, Lille, France, 6-11 July 2015; pp. 2048-2057.

34. Luong, M.T.; Pham, H.; Manning, C.D. Effective approaches to attention-based neural machine translation. arXiv 2015, arXiv:1508.04025.

35. Bahdanau, D.; Cho, K.; Bengio, Y. Neural machine translation by jointly learning to align and translate. arXiv 2014, arXiv:1409.0473.

36. Song, H.; Rajan, D.; Thiagarajan, J.J.; Spanias, A. Attend and Diagnose: Clinical Time Series Analysis Using Attention Models. In Proceedings of the Thirty-second AAAI conference on artificial intelligence, New Orleans, LA, USA, 2-7 February 2018.

37. Chaudhari, S.; Polatkan, G.; Ramanath, R.; Mithal, V. An attentive survey of attention models. arXiv 2019, arXiv:1904.02874. 
38. Raffel, C.; Ellis, D.P. Feed-forward networks with attention can solve some long-term memory problems. arXiv 2015, arXiv:1512.08756.

39. Shi, B.; Bai, X.; Yao, C. An end-to-end trainable neural network for image-based sequence recognition and its application to scene text recognition. IEEE Trans. Pattern Anal. Mach. Intell. 2016, 39, 2298-2304. [CrossRef]

40. Oh, S.L.; Ng, E.Y.; San Tan, R.; Acharya, U.R. Automated diagnosis of arrhythmia using combination of CNN and LSTM techniques with variable length heart beats. Comput. Biol. Med. 2018, 102, 278-287. [CrossRef] [PubMed]

41. Kim, Y.; Yeo, M.; Sohn, I.; Park, C. Multimodal drowsiness detection methods using machine learning algorithms. IEIE Trans. Smart Process. Comput. 2018, 7, 361-365 [CrossRef]

42. Seok, W.; Park, C. Recognition of Human Motion with Deep Reinforcement Learning. IEIE Trans. Smart Process. Comput. 2018, 7, 245-250 [CrossRef]

43. Vaswani, A.; Shazeer, N.; Parmar, N.; Uszkoreit, J.; Jones, L.; Gomez, A.N.; Kaiser, Ł.; Polosukhin, I. Attention Is All You Need. In Proceedings of the Advances in neural information processing systems, Long Beach, CA, USA, 4-9 December 2017; pp. 5998-6008.

44. Simonyan, K.; Zisserman, A. Very deep convolutional networks for large-scale image recognition. arXiv 2014, arXiv:1409.1556.

45. Ioffe, S.; Szegedy, C. Batch normalization: Accelerating deep network training by reducing internal covariate shift. arXiv 2015, arXiv:1502.03167.

46. Kingma, D.P.; Ba, J. Adam: A method for stochastic optimization. arXiv 2014, arXiv:1412.6980.

47. Altman, D.G.; Bland, J.M. Measurement in medicine: the analysis of method comparison studies. J. R. Stat. Soc. Ser. D 1983, 32, 307-317. [CrossRef]

48. Pan, J.; Tompkins, W.J. A real-time QRS detection algorithm. IEEE Trans. Biomed. Eng. 1985, $230-236$. [CrossRef]

49. Association for the Advancement of Medical Instrumentation. American National Standards for Electronic or automated sphygmomanometers. ANSI AAMI 1992, 1-40.

50. O'Brien, E.; Petrie, J.; Littler, W.; de Swiet, M.; Padfield, P.L.; Altman, D.; Bland, M.; Coats, A.; Atkins, N. The British Hypertension Society protocol for the evaluation of blood pressure measuring devices. J. Hypertens. 1993, 11, S43-S62.

51. Whelton, P.K.; Carey, R.M. The 2017 American College of Cardiology/American Heart Association clinical practice guideline for high blood pressure in adults. JAMA Cardiol. 2018, 3, 352-353. [CrossRef] [PubMed]

(C) 2020 by the authors. Licensee MDPI, Basel, Switzerland. This article is an open access article distributed under the terms and conditions of the Creative Commons Attribution (CC BY) license (http:/ / creativecommons.org/licenses/by/4.0/). 